Supporting Information

\title{
Crystal Engineering of a Hydrazone Molecule toward High Elasticity and Bright Luminescence
}

\author{
Bin Liu, Hao Liu, Houyu Zhang, Qi Di, Hongyu Zhang*
}

State Key Laboratory of Supramolecular Structure and Materials, College of Chemistry, Jilin

University, Qianjin Street, Changchun, 130012, P. R. China. E-mail:hongyuzhang@jlu.edu.cn

\section{Contents}

1. General information $\quad$ S2

2. Synthetic details S2-S3

3. X-ray crystallographic analysis S4-S6

4. Growth morphology calculation of single crystal S6-S8

5. TD-DFT calculation S9-S10

6. Optical waveguide properties S11-S12

$\begin{array}{ll}\text { 7. Thermal properties } & \mathbf{S 1 2}\end{array}$ 


\section{General information}

Commercially available solvents and reagents were used without further purification unless otherwise mentioned. ${ }^{1} \mathrm{H}$ and ${ }^{13} \mathrm{C}\left\{{ }^{1} \mathrm{H}\right\}$ NMR spectra were recorded with a Bruker Avance $500 \mathrm{MHz}$ spectrometer (500 MHz for ${ }^{1} \mathrm{H}$ and $126 \mathrm{MHz}$ for ${ }^{13} \mathrm{C}$ ) in DMSO- $d_{6}$. The chemical shifts in ${ }^{1} \mathrm{H}$ NMR and ${ }^{13} \mathrm{C}$ NMR spectra are reported in $\delta$ ppm using tetramethylsilane as an internal standard. Mass spectra were recorded on a Thermo Fisher ITQ1100 mass spectrometer. Element analyses were performed on a Flash EA1112 spectrometer. The crystal slice was irradiated by the third harmonic $(355 \mathrm{~nm})$ of a Nd:YAG (yttrium-aluminum-garnet) laser at a repetition rate of $10 \mathrm{~Hz}$ and a pulse duration of about 10 ns. The energy of laser was adjusted by using the calibrated neutral density filters. The beam was focused into a stripe whose shape was adjusted to $3.3 \times 0.6 \mathrm{~mm}$ by using a cylindrical lens and a slit. The emission was detected at one end of the crystal using a Maya2000 Pro CCD spectrometer. Differential scanning calorimetric (DSC) measurements were performed on a NETZSCH DSC204 instrument at a heating rate of $10{ }^{\circ} \mathrm{C} \mathrm{min}^{-1}$ under nitrogen.

\section{Synthetic details}

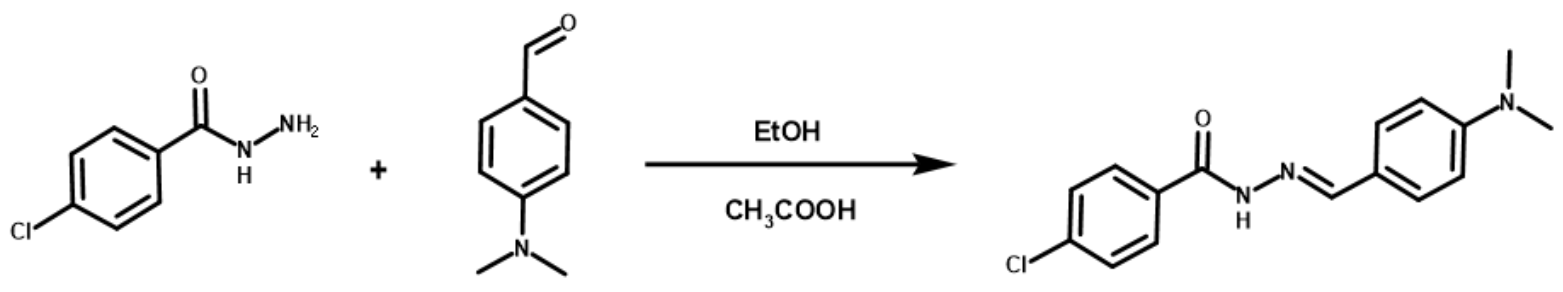

Scheme S1. The synthetic procedure of DBBH.

$1.701 \mathrm{~g}$ 4-chlorobenzohydrazide (10.0 mmol), $1.492 \mathrm{~g} \mathrm{4-(dimethylamino)benzaldehyde} \mathrm{(10.0} \mathrm{mmol)}$ and $60 \mathrm{ml}$ ethanol were mixed and stirred for $0.5 \mathrm{~h}$. After cooling to room temperature, a green precipitate was obtained and collected by filtration, washed with ethanol and dried in vacuum to afford probe DBBH as a green powder (85\% yield, $2.565 \mathrm{~g}$ ). ${ }^{1} \mathrm{H}$ NMR (500 MHz, DMSO- $d 6$ ): $\delta 11.61$ (s, 1H), 8.30 (s, 1H), 7.92 (d, J = 8.7 Hz, 2H), 7.59 (d, J = 8.3 Hz, 2H), 7.54 (d, J = 8.6 Hz, 2H), 6.76 (d, J $=8.8 \mathrm{~Hz}, 2 \mathrm{H}), 2.98(\mathrm{~s}, 6 \mathrm{H}) \cdot{ }^{13} \mathrm{C}\left\{{ }^{1} \mathrm{H}\right\} \mathrm{NMR}(126 \mathrm{MHz}, \mathrm{DMSO}-d 6): \delta$ 162.01, 152.03, 149.41, 136.70, 132.98, 129.88, 128.95, 121.93, 112.26, 40.23. MS m/z: 301.45 [M] ${ }^{+}$(calcd: 301.77). Anal. calcd (\%) for $\mathrm{C}_{16} \mathrm{H}_{16} \mathrm{ClN}_{3} \mathrm{O}: \mathrm{C}, 63.68 ; \mathrm{H}, 5.34 ; \mathrm{Cl}, 11.75 ; \mathrm{N}, 13.92 ; \mathrm{O}, 5.30$. Found:C, 68.71; H, 5.36; N, 13.89 . 


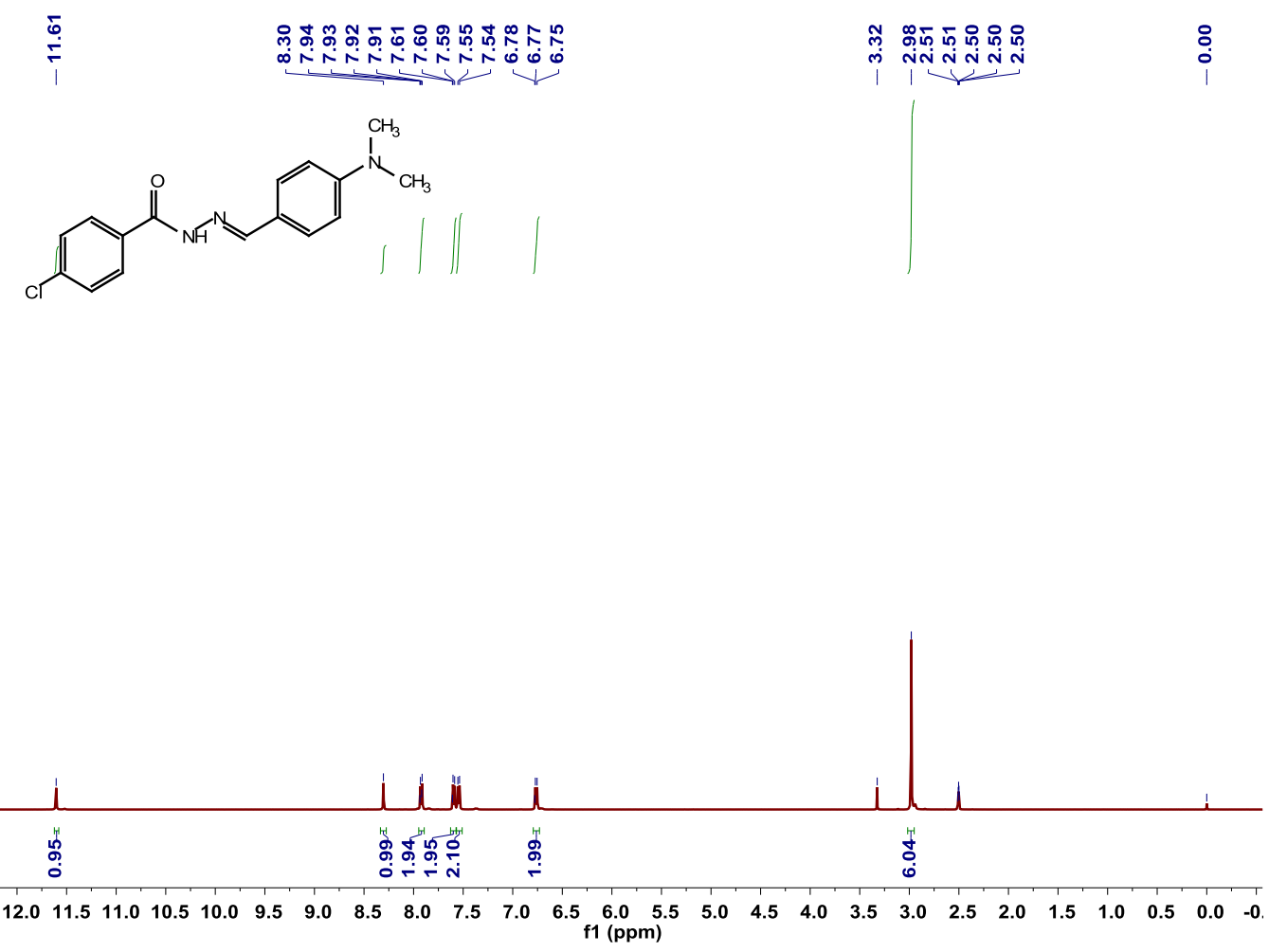

Figure S1. ${ }^{1} \mathrm{H}$ NMR spectrum of DBBH $\left(500 \mathrm{MHz}\right.$, DMSO- $\left.d_{6}\right)$
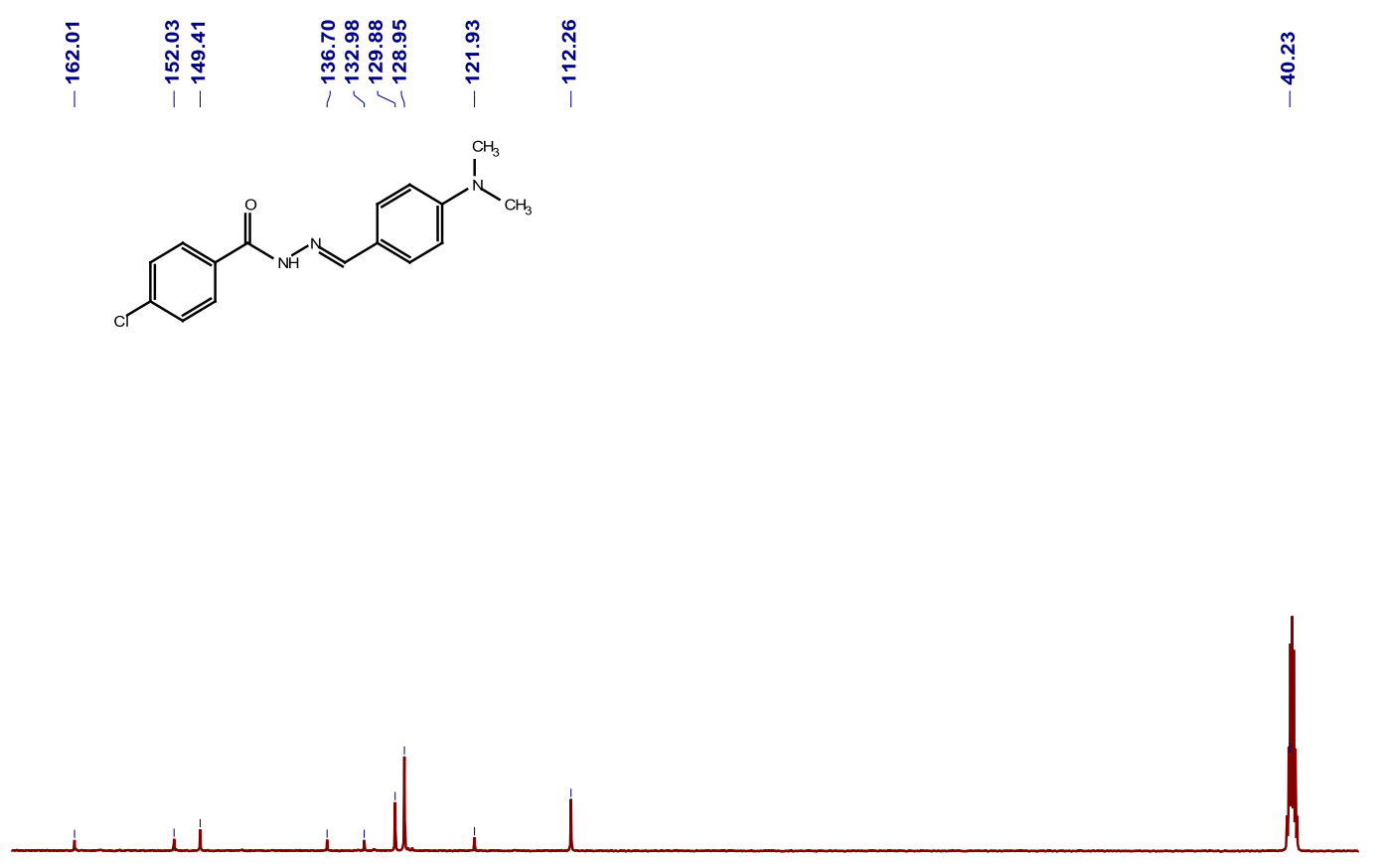

$\begin{array}{llllllllllllllllllllllllllllllllllll}165 & 160 & 155 & 150 & 145 & 140 & 135 & 130 & 125 & 120 & 115 & 110 & 105 & 100 & 95 & 90 & 85 & 80 & 75 & 70 & 65 & 60 & 55 & 50 & 45 & 40 & 35\end{array}$

Figure S2. ${ }^{13} \mathrm{C}\left\{{ }^{1} \mathrm{H}\right\}$ NMR spectrum of DBBH $\left(126 \mathrm{MHz}, \mathrm{DMSO}-d_{6}\right)$. 


\section{X-ray crystallographic analysis}

Powder X-ray diffraction measurements. Powder X-ray diffraction data of five crystals were collected on a Rigaku SmartLab 3 diffractometer with $\mathrm{Cu} \cdot \mathrm{K} \alpha$ radiation.

Single crystal X-ray measurements. Single crystal X-ray diffraction data were collected on a Rigaku RAXIS-PRID diffractometer using the $\omega$-scan mode with graphite monochromator Mo $\bullet \alpha$ radiation. The structures were solved with direct methods using the SHELXTL programs and refined with full-matrix least-squares on $F^{2}$. Non-hydrogen atoms were refined anisotropically. The positions of hydrogen atoms were calculated and refined isotropically. The crystallographic information has been deposited at the Cambridge Crystallographic Data Centre (CCDC).

CCDC number: Cry-A: 2024538 Cry-B:2024541 Cry-C: 2024537 Cry-D: 2024540 Cry-E: 2024539

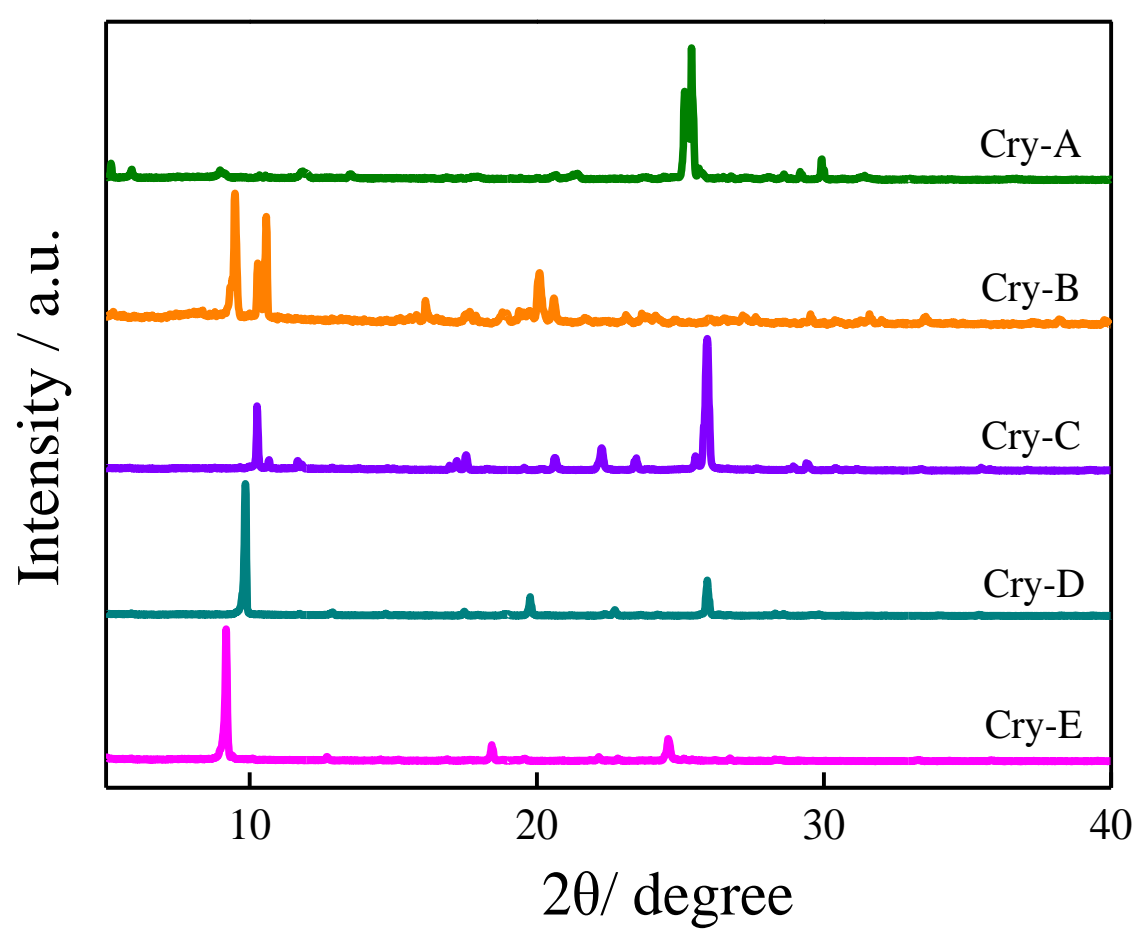

Figure S3. The measured (XRD) patterns of Cry-A, B, C, D and E. 
Table S1. Crystal data and structural refinement for the crystals of DBBH.

\begin{tabular}{|c|c|c|c|c|c|}
\hline Identification code & Cry-A & Cry-B & Cry-C & Cry-D & Cry-E \\
\hline Empirical formula & $\mathrm{C}_{16} \mathrm{H}_{16} \mathrm{CIN}_{3} \mathrm{O}$ & $\mathrm{C}_{32} \mathrm{H}_{32} \mathrm{Cl}_{2} \mathrm{~N}_{6} \mathrm{O}_{2}$ & $\mathrm{C}_{17} \mathrm{H}_{20} \mathrm{ClN}_{3} \mathrm{O}_{2}$ & $\mathrm{C}_{18} \mathrm{H}_{22} \mathrm{CIN}_{3} \mathrm{O}_{2}$ & $\mathrm{C}_{19} \mathrm{H}_{24} \mathrm{ClN}_{3} \mathrm{O}_{2}$ \\
\hline Formula weight & 301.77 & 603.54 & 333.81 & 347.84 & 361.86 \\
\hline Temperature/K & 297.8 & 297.8 & 297.8 & 297.8 & 297.8 \\
\hline Crystal system & monoclinic & orthorhombic & monoclinic & monoclinic & monoclinic \\
\hline Space group & $\mathrm{P} 2 / \mathrm{c}$ & Pbca & $\mathrm{P} 2{ }_{1} / \mathrm{c}$ & $\mathrm{P} 2_{1} / \mathrm{c}$ & $\mathrm{P} 2 / \mathrm{c}$ \\
\hline $\mathrm{a} / \AA ̊ \AA$ & $15.1980(9)$ & $11.3576(10)$ & $15.5657(8)$ & $15.7804(8)$ & $15.7416(9)$ \\
\hline $\mathrm{b} / \AA \AA$ & $13.1294(8)$ & $8.0121(6)$ & $6.4012(3)$ & $6.3813(3)$ & $6.4349(4)$ \\
\hline$c / \AA ̊$ & $7.7881(5)$ & $67.179(6)$ & $17.6685(9)$ & $18.8376(10)$ & $20.1152(12)$ \\
\hline$\alpha /^{\circ}$ & 90.00 & 90.00 & 90.00 & 90.00 & 90.00 \\
\hline$\beta /^{\circ}$ & $98.169(3)$ & 90.00 & $102.526(2)$ & $107.348(2)$ & $106.323(3)$ \\
\hline $\mathrm{y}^{\circ}$ & 90.00 & 90.00 & 90.00 & 90.00 & 90.00 \\
\hline Volume $/ \AA^{3}$ & $1538.27(16)$ & $6113.2(9)$ & $1718.57(15)$ & $1810.65(16)$ & $1955.5(2)$ \\
\hline Z & 4 & 8 & 4 & 4 & 4 \\
\hline$\rho_{\text {calc }} \mathrm{g} / \mathrm{cm}^{3}$ & 1.303 & 1.312 & 1.290 & 1.276 & 1.229 \\
\hline$\mu / \mathrm{mm}^{-1}$ & 0.250 & 0.252 & 0.235 & 0.226 & 0.212 \\
\hline$F(000)$ & 632.0 & 2528 & 704 & 736 & 768 \\
\hline Radiation & $\begin{array}{l}\operatorname{MoKa}(\lambda= \\
0.71073)\end{array}$ & $\begin{array}{l}\text { MoKa }(\lambda= \\
0.71073)\end{array}$ & $\begin{array}{l}\text { MoKa }(\lambda= \\
0.71073)\end{array}$ & $\operatorname{MoKa}(\lambda=0.71073)$ & $\begin{array}{l}\text { MoKa }(\lambda= \\
0.71073)\end{array}$ \\
\hline $\begin{array}{c}2 \Theta \text { range for data } \\
\text { collection }^{\circ}\end{array}$ & 5.42 to 50 & 6.066 to 52.542 & 5.36 to 52.74 & 5.4 to 52.82 & 5.4 to 48.98 \\
\hline Index ranges & $\begin{array}{c}-18 \leq h \leq 17,-15 \leq \\
k \leq 15,-9 \leq 1 \leq 9\end{array}$ & $\begin{array}{c}-13 \leq h \leq 13,-9 \leq k \\
\leq 9,-79 \leq 1 \leq 79\end{array}$ & $\begin{array}{c}-19 \leq \mathrm{h} \leq 19,-7 \leq \mathrm{k} \\
\leq 8,-22 \leq 1 \leq 22\end{array}$ & $\begin{array}{c}-19 \leq h \leq 19,-7 \leq k \leq \\
7,-23 \leq 1 \leq 23\end{array}$ & $\begin{array}{l}-18 \leq h \leq 18,-7 \leq \\
k \leq 7,-23 \leq 1 \leq 23\end{array}$ \\
\hline Reflections collected & 19414 & 84796 & 42001 & 22010 & 25128 \\
\hline Independent reflections & $\begin{array}{c}2615\left[R_{\text {int }}=0.0793,\right. \\
\left.R_{\text {sigma }}=0.0501\right]\end{array}$ & $\begin{array}{c}5390\left[R_{\text {int }}=\right. \\
0.0643, R_{\text {sigma }}= \\
0.0234]\end{array}$ & $\begin{array}{c}3498\left[R_{\text {int }}=\right. \\
0.0921, R_{\text {sigma }}= \\
0.0391]\end{array}$ & $\begin{array}{c}3700\left[R_{\text {int }}=0.0650\right. \\
\left.R_{\text {sigma }}=0.0432\right]\end{array}$ & $\begin{array}{c}3092\left[R_{\text {int }}=\right. \\
0.0931, R_{\text {sigma }}= \\
0.0572]\end{array}$ \\
\hline Data/restraints/parameters & $2615 / 0 / 193$ & $5390 / 0 / 383$ & $3498 / 0 / 213$ & $3700 / 0 / 232$ & $3700 / 1 / 230$ \\
\hline Goodness-of-fit on $\mathrm{F}^{2}$ & 1.045 & 1.246 & 1.016 & 1.022 & 1.162 \\
\hline Final $R$ indexes $[\mid>=2 \sigma(I)]$ & $\begin{array}{c}\mathrm{R}_{1}=0.0668, \mathrm{wR} \mathrm{R}_{2}= \\
0.1495\end{array}$ & $\begin{array}{c}\mathrm{R}_{1}=0.0790, w \mathrm{R}_{2}= \\
0.1588\end{array}$ & $\begin{aligned} \mathrm{R}_{1}= & 0.0480, \mathrm{wR} \mathrm{R}_{2} \\
& =0.0972\end{aligned}$ & $\begin{aligned} \mathrm{R}_{1}= & 0.0464, w \mathrm{R}_{2} \\
= & 0.0995\end{aligned}$ & $\begin{aligned} R_{1}= & 0.0983, w R_{2} \\
& =0.2027\end{aligned}$ \\
\hline
\end{tabular}




\begin{tabular}{|c|c|c|c|c|c|}
\hline Final $R$ indexes [all data] & $\begin{array}{c}\mathrm{R}_{1}=0.1310, \mathrm{wR}_{2}= \\
0.1902\end{array}$ & $\begin{array}{c}\mathrm{R}_{1}=0.0839, \mathrm{wR} \mathrm{R}_{2}= \\
0.1607\end{array}$ & $\begin{array}{c}\mathrm{R}_{1}=0.1001, \mathrm{wR}_{2} \\
=0.1214\end{array}$ & $\begin{array}{c}\mathrm{R}_{1}=0.0955, \mathrm{wR}_{2} \\
=0.1247\end{array}$ & $\begin{array}{c}\mathrm{R}_{1}=0.1630, \mathrm{wR} \mathrm{R}_{2} \\
=0.2380\end{array}$ \\
\hline $\begin{array}{c}\text { Largest diff. peak/hole / } \mathrm{e} \\
\AA^{-3}\end{array}$ & $0.24 /-0.20$ & $0.29 /-0.40$ & $0.17 /-0.17$ & $0.18 /-0.22$ & $0.48 /-0.29$ \\
\hline
\end{tabular}

\section{Growth morphology calculation of single crystal}

The growth morphology of CN-DPVB and its hydrochloride crystals were calculated by the software of Materials Studio 8.0 with the crystallographic data as the input information. In general, the growth rate of a crystalline plane is assumed to be proportional to its attachment energy $\left(\mathrm{E}_{\text {att }}\right)$. The plane with lower attachment energy is slower growing and hence has more morphological importance. The attachment energy can be calculated: $E_{\text {att }}=E_{\text {lattice }}-E_{\text {slice}}$, where $E_{\text {lattice }}$ is the lattice energy of the crystal and $E_{\text {slice }}$ is the energy for a growth slice of thickness $d_{\text {hkl }}$

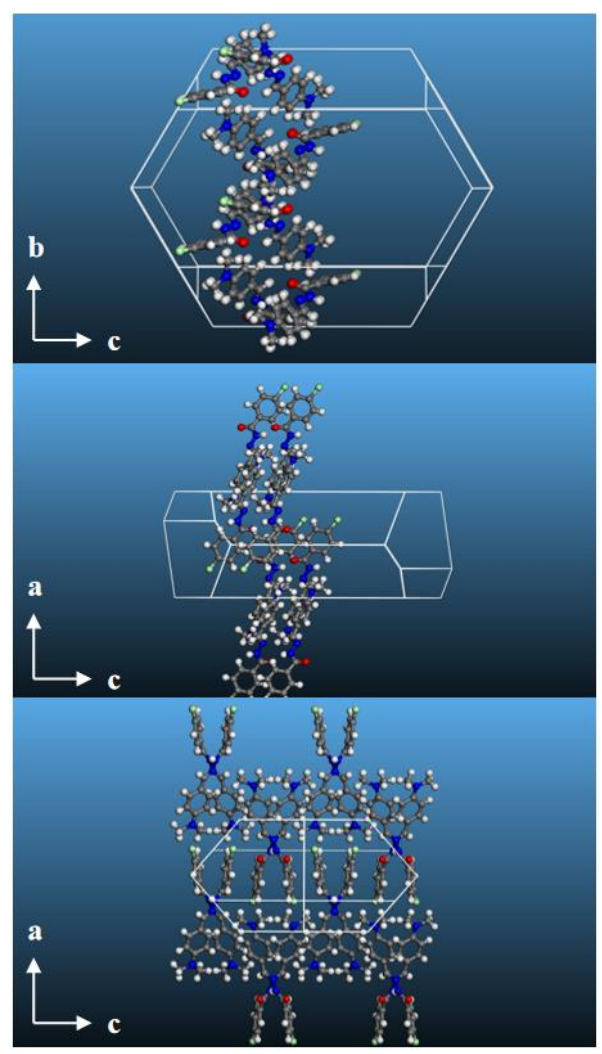

Figure S4. Calculated growth morphology of Cry-A. 


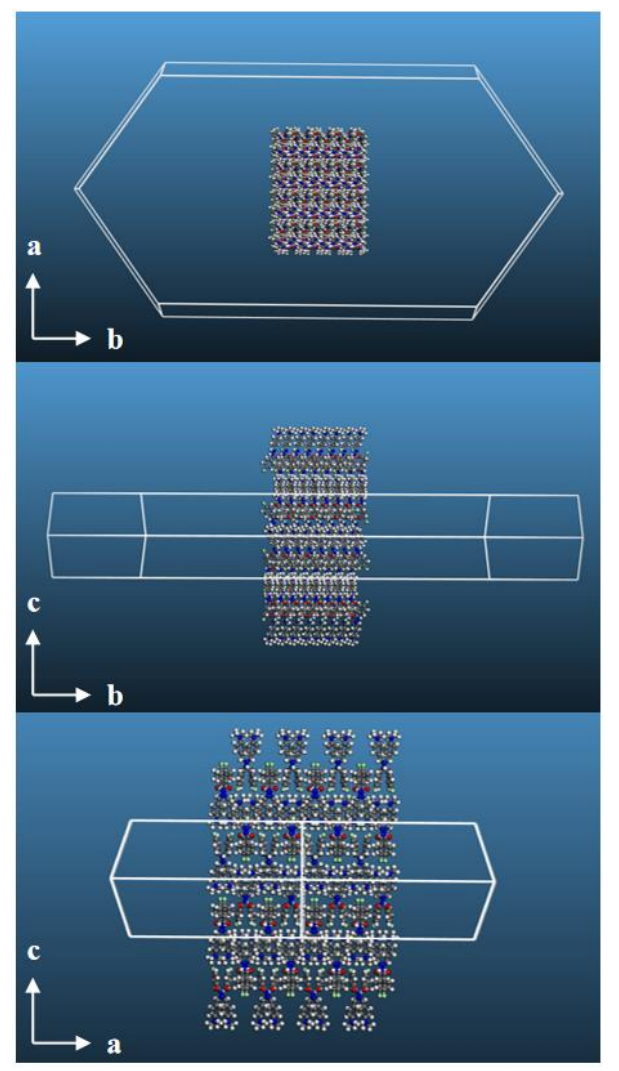

Figure S5. Calculated growth morphology of Cry-B.

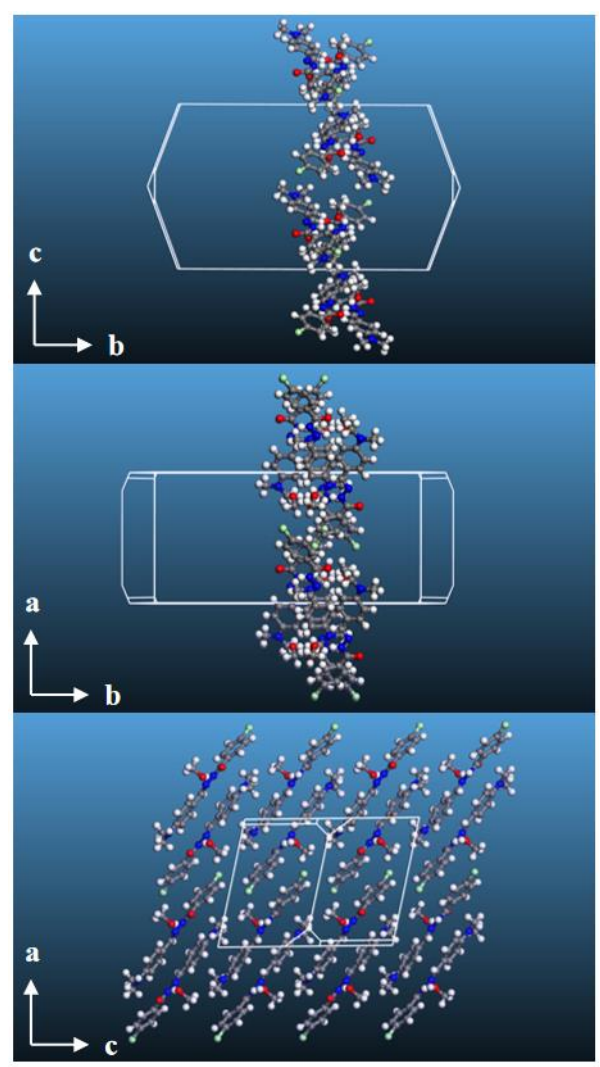

Figure S6. Calculated growth morphology of Cry-C. 


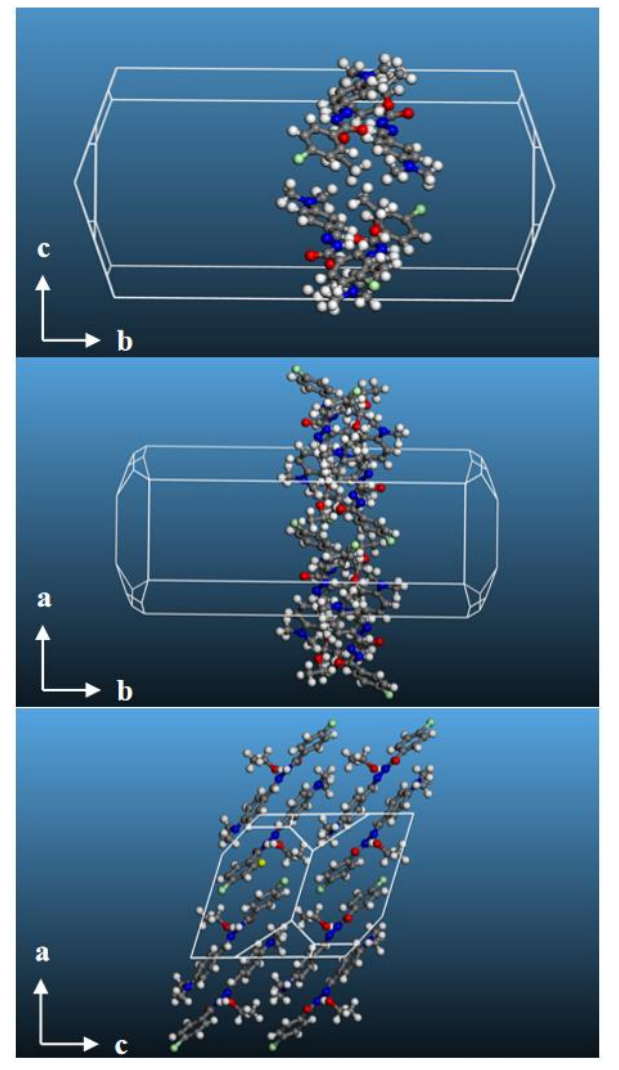

Figure S7. Calculated growth morphology of Cry-D.
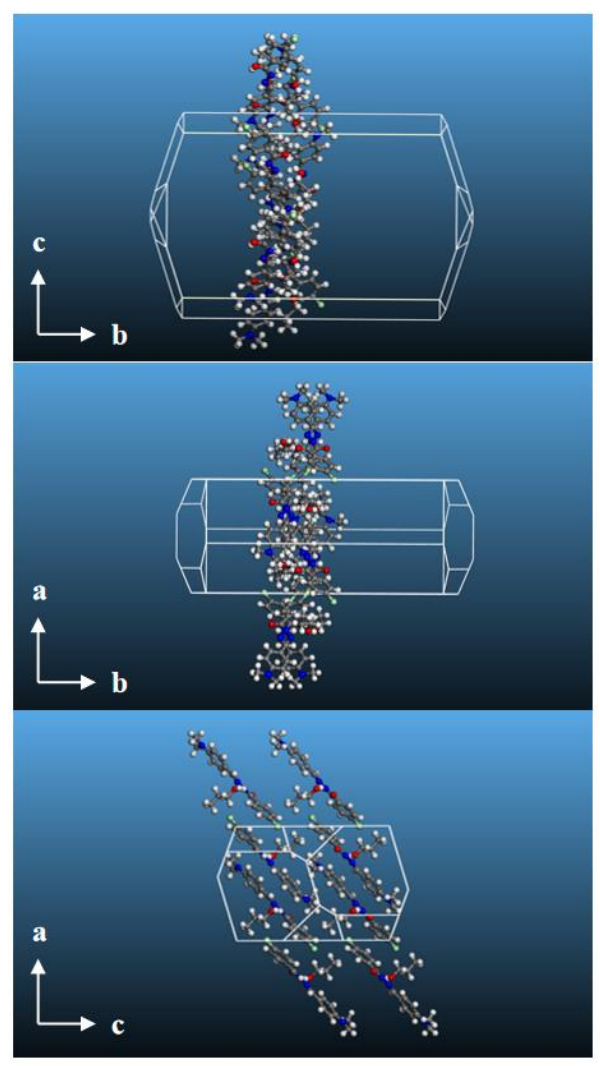

Figure S8. Calculated growth morphology of Cry-E. 


\section{TD-DFT calculation}

Computational details: TD-DFT calculation was performed using the B3LYP hybrid functional with 6-31G(d, p) basis set. To simulate the spectra, electronic excitations were calculated by iteratively solving 50 states. To describe the intermolecular interaction accurately, semiempirical dispersion was included by using D3 version of Grimme' s dispersion. All calculation carried out with the G09 package.

A
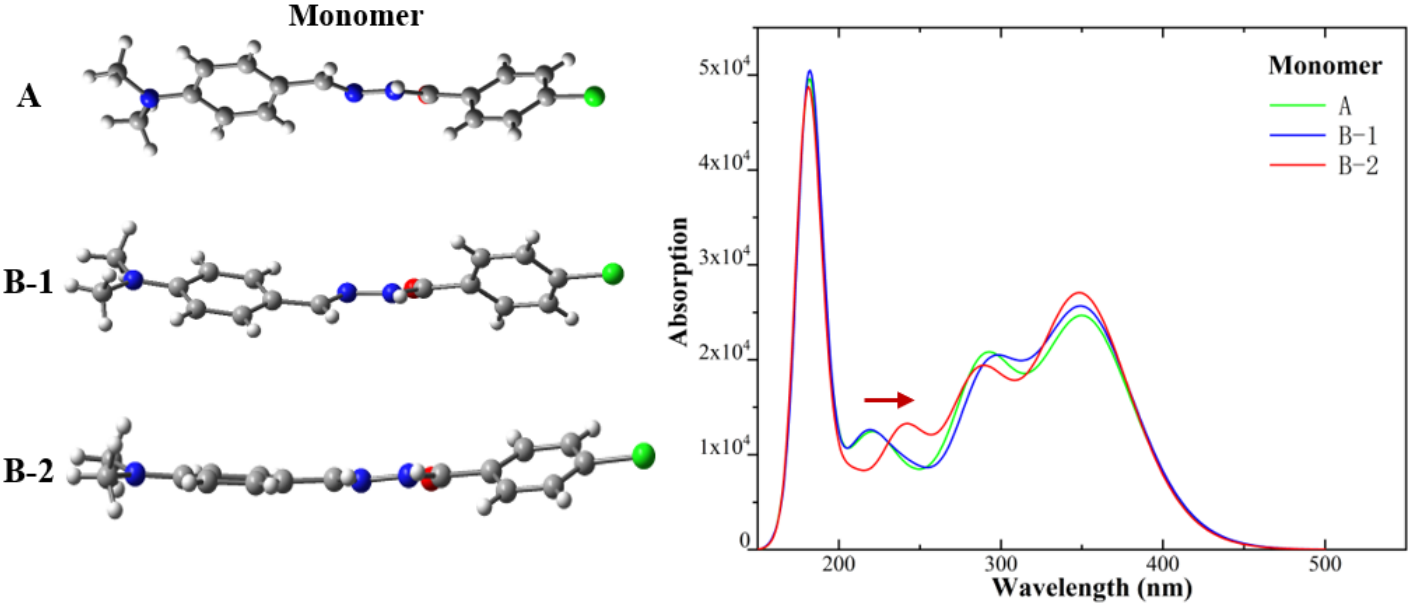

Figure S9. Monomer structures and absorption spectra of Cry-A and Cry-B.

$\mathbf{A}$
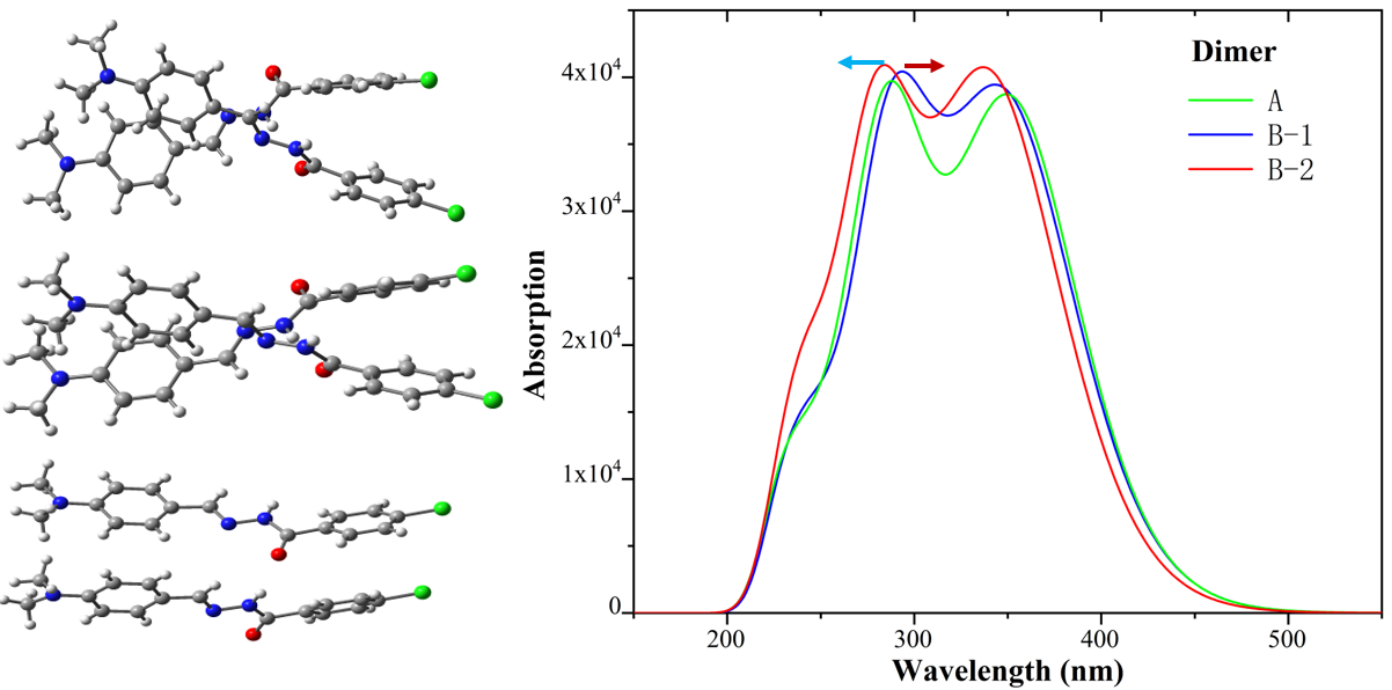

Figure S10. Dimer structures and absorption spectra of Cry-A and Cry-B. 

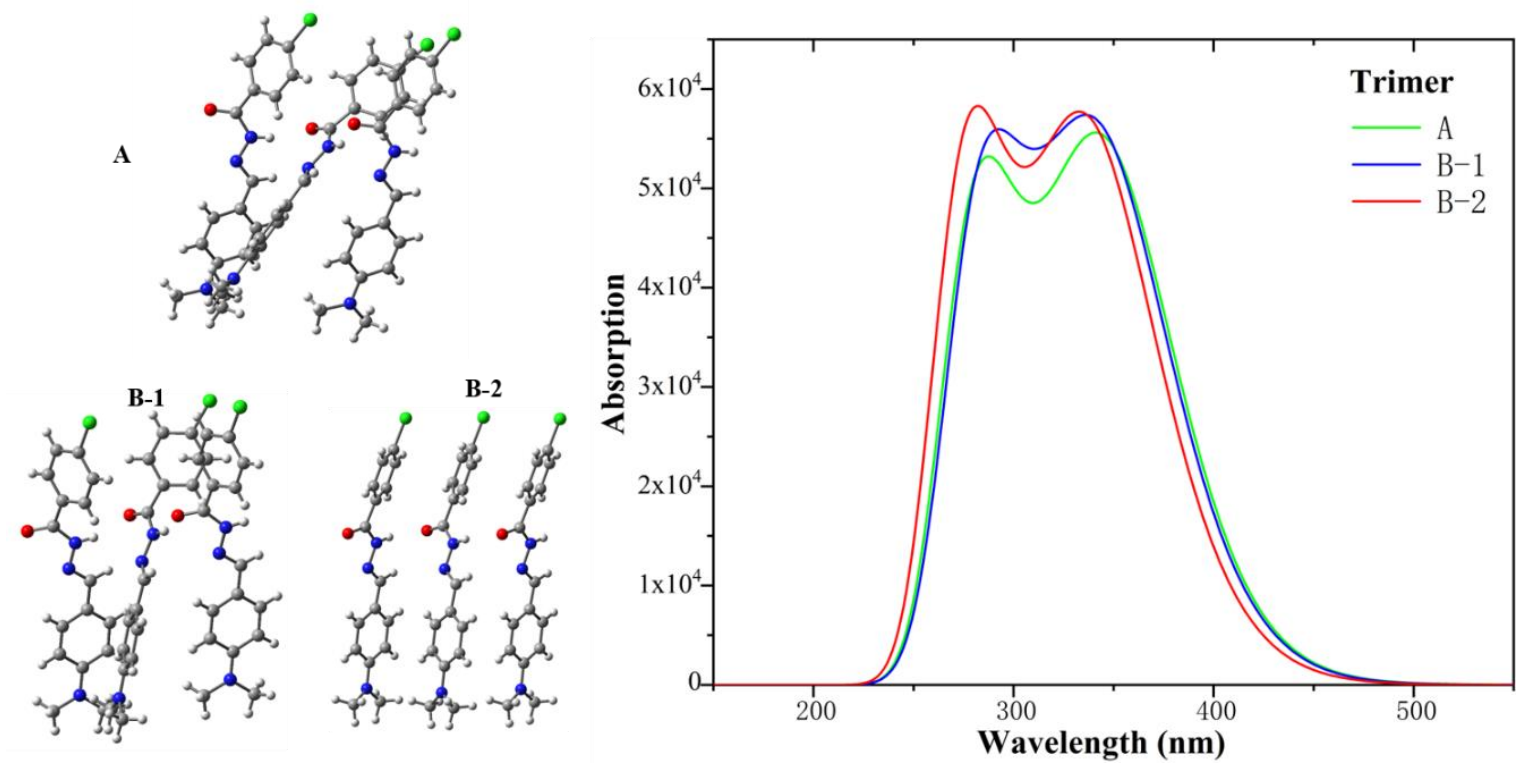

Figure S11. Trimer structures and absorption spectra of Cry-A and Cry-B.

Table S2. Absorption peak and peak-to-peak separation in dimer and trimer of crystals.

\begin{tabular}{l|l|l|l|l|l|l}
\hline & \multicolumn{2}{c}{ A } & \multicolumn{2}{c}{ B-1 } & \multicolumn{2}{c}{ B-2 } \\
\cline { 2 - 7 } & Dimer & Trimer & Dimer & trimer & Dimer & Trimer \\
\hline Peak 1 & 288 & 287 & 294 & 294 & 284 & 282 \\
\hline Peak 2 & 348 & 341.6 & 343 & 336 & 337.4 & 33.2 \\
\hline peak-to-peak & 60 & 54.6 & 49 & 42 & 53.4 & 51.2 \\
separation & & & & & & \\
\hline
\end{tabular}




\section{Optical waveguide properties}
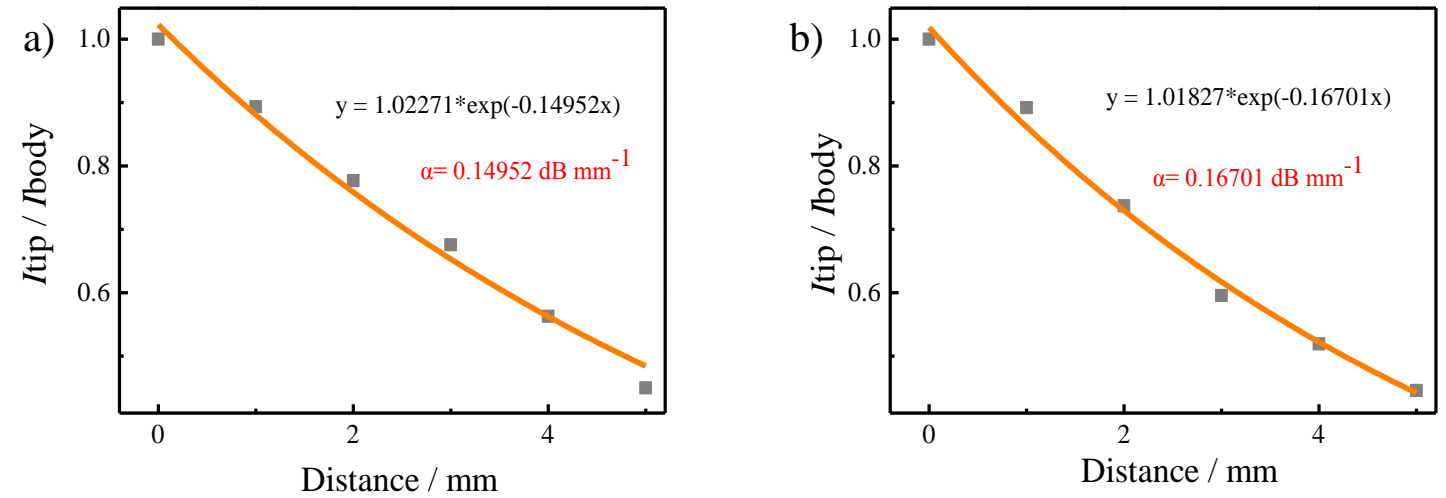

Figure S12. The $I_{\text {tip }} / I_{\text {body }}$ decays of the Cry-D in the straight (a) and naturally bent (b) states. The optical loss coefficients $(\alpha)$ are attained by a single exponential fitting of the function $I_{\text {tip }} / I_{\text {body }}=$ Aexp(- $\alpha \mathrm{D})$, where $I_{\text {tip }}$ and $I_{\text {body }}$ are the fluorescence intensities of out-coupled and incidence light and $\mathrm{D}$ is the distance between the excited site and the tip for collecting emission.
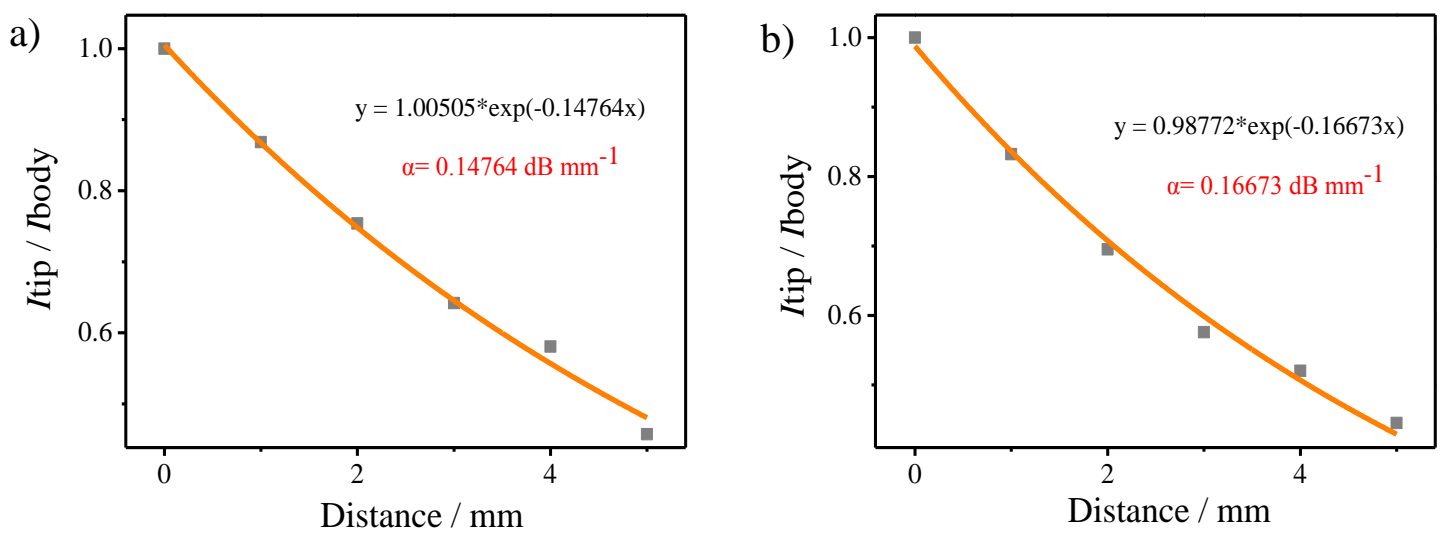

Figure S13. The $I_{\text {tip }} / I_{\text {body }}$ decays of the Cry-C in the straight (a) and naturally bent (b) states. The optical loss coefficients $(\alpha)$ are attained by a single exponential fitting of the function $I_{\text {tip }} / I_{\text {body }}=$ Aexp(- $\alpha \mathrm{D})$, where $I_{\text {tip }}$ and $I_{\text {body }}$ are the fluorescence intensities of out-coupled and incidence light and $\mathrm{D}$ is the distance between the excited site and the tip for collecting emission. 

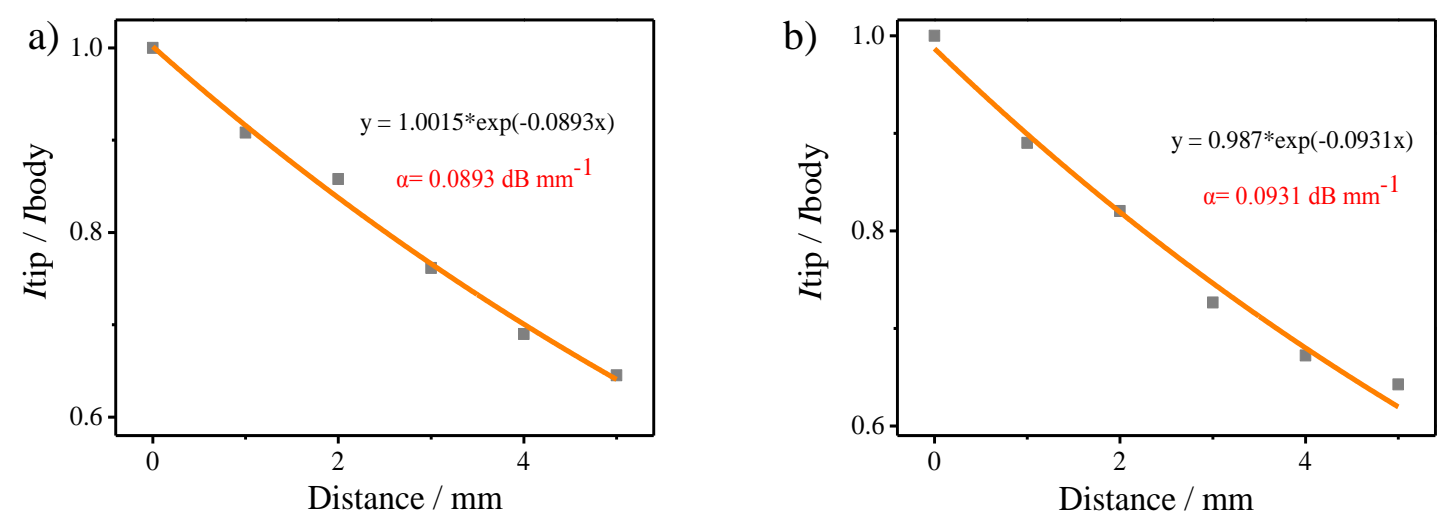

Figure S14. The $I_{\text {tip }} / I_{\text {body }}$ decays of the Cry-E in the straight (a) and naturally bent (b) states. The optical loss coefficients $(\alpha)$ are attained by a single exponential fitting of the function $I_{\text {tip }} / I_{\text {body }}=$ Aexp(- $\alpha \mathrm{D})$, where $I_{\text {tip }}$ and $I_{\text {body }}$ are the fluorescence intensities of out-coupled and incidence light and $\mathrm{D}$ is the distance between the excited site and the tip for collecting emission.

\section{Thermal properties}

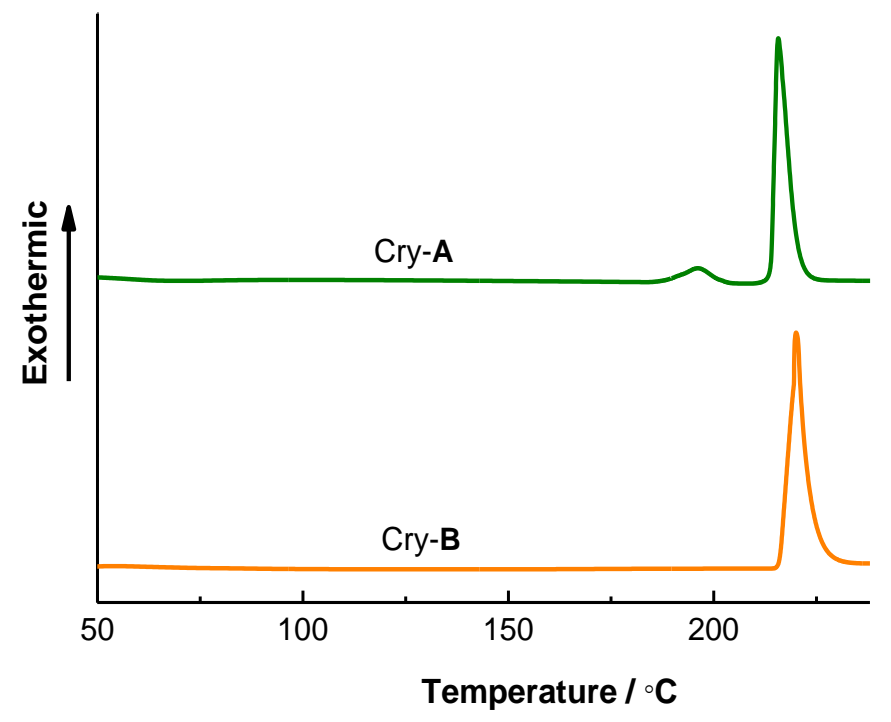

Figure S15. The differential scanning calorimetric (DSC) curves of Cry-A and Cry-B. 